\title{
Non-Contact Spin Pumping by Microwave Evanescent Fields
}

\author{
Tao $\mathrm{Yu}^{1}$ and Gerrit E. W. Bauer ${ }^{2,1}$ \\ ${ }^{1}$ Kavli Institute of NanoScience, Delft University of Technology, 2628 CJ Delft, The Netherlands \\ ${ }^{2}$ Institute for Materials Research \&S WPI-AIMR \& CSRN, Tohoku University, Sendai 980-8577, Japan
}

(Dated: July 29, 2020)

\begin{abstract}
The angular momentum of evanescent light fields has been studied in nano-optics and plasmonics, but not in the microwave regime. Here we predict non-contact pumping of electron spin currents in conductors by the evanescent stray fields of excited magnetic nanostructures. The coherent transfer of the photon to the electron spin is proportional to the $g$-factor, which is large in narrow-gap semiconductors and surface states of topological insulators. The spin pumping current is chiral when the spin susceptibility displays singularities that indicate collective states. However, $1 \mathrm{D}$ systems with linear dispersion at the Fermi energy such as metallic carbon nanotubes are an exception since spin pumping is chiral even without interactions.
\end{abstract}

Introduction.-Efficient transfer of spin information among different entities is a key objective in spintronics 1]. The electromagnetic field at frequency $\omega$ carries a spin angular momentum density [2, 3]

$$
\mathcal{D}=\frac{1}{4 \omega} \operatorname{Im}\left(\varepsilon_{0} \mathbf{E}^{*} \times \mathbf{E}+\mu_{0} \mathbf{H}^{*} \times \mathbf{H}\right),
$$

where $\mu_{0} / \varepsilon_{0}$ are the vacuum permeability/permittivity, and in the microwave regime the magnetic field component $\sim \operatorname{Im}\left(\mathbf{H}^{*} \times \mathbf{H}\right)$ dominates the contribution of the electric field $\mathbf{E}$. The evanescent fields at boundaries can have local angular momentum even when the (linearlypolarized) propagating ones have not [4, 5. A distinguishing feature of such evanescent fields is the locking between the linear and angular momentum [2, 3, 6, 7. The chiral electrical near-field of a rotating electrical dipole [4, 5], e.g., unidirectionally excites surface plasmon polaritons [5, 6, 8. Metallic striplines or coplanar waveguides biased by currents in the $\mathrm{GHz}$ regime also emit chiral magnetic near-fields 9-11, which is of considerable interest for magnonics [12 15, since chiral excitation is a robust and switchable mechanism to pump a DC unidirectional magnon current by an AC field [16, 17.

Spin pumping by exchange interaction is established when the magnet and conductor form a good electric contact, which is difficult to achieve between metals and semiconductors including graphene because of Schottky barriers and electronic structure mismatch [18, 19]. Even when a good contact to a magnet can be established, results may be difficult to interpret due to proximity effects. Spin pumping at a distance by microwaves solves these issues since it does not require direct contact between the magnet and the system of interest. In this Letter, we address the non-contact angular momentum transfer to an electric conductor by stray magnetic fields emitted by an excited magnet, thereby generalizing the concept of spin pumping by a contact exchange interaction [18, 19]. We are motivated by the significant near fields that couple magnetic nanowires and ultrathin magnetic insulating films, causing several chiral magnon transport phenomena [17, 20, 26]. Here we demonstrate that a magnetodipolar field pumps electron spins into a conductor without need of electric contacts. We illustrate the physics for a simple yet realistic model system of a magnetic nanowire on top of a two-dimensional electron gas (2DEG) as illustrated in Fig. 1. The latter may be graphene 27 30, but the effect is strongly enhanced by spin-orbit interaction, such as a large $g$-factor in InAs or InSb quantum wells (QWs) [31, 32] or the surface states of 3D topological insulator [33, 34]. In contrast to the dipolar spin pumping of magnons, the spin pumping current in non-interacting conductors is in general not chiral. However, the singular spin susceptibility in onedimensional systems with linear dispersion at the Fermi energy such as metallic carbon nanotubes generates chirality of the spin injection with and without interactions.

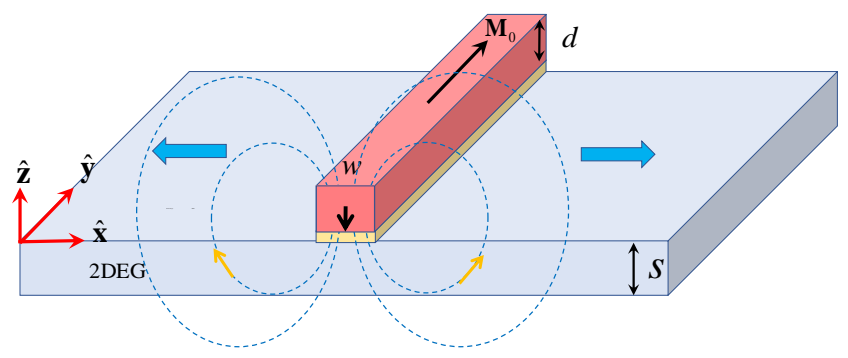

FIG. 1. Snapshot of spin pumping by the microwave dipolar field of an excited magnetic nanowire on top of a 2DEG. A thin tunneling barrier suppresses any exchange coupling. Orange arrows indicate the direction of stray field.

Transverse spin density of microwaves.-We first demonstrate that the evanescent magnetodipolar field of a magnetic nanowire carries transverse angular momentum or "spin". The nanowire with width $w$, thickness $d$ and equilibrium magnetization $\mathbf{M}_{s}$ along the wire $y$ direction, on top of an electron gas confined in the $z$ direction on a length scale $s$ as illustrated in Fig. 1) acts as an antenna for external microwaves with frequency tuned to the ferromagnetic resonance (FMR) $\omega_{K}$ [21, 35, 36]. In the following we use a quantum mechan- 
ical notation for convenience, but in the classical limit operators can be simply replaced by field amplitudes. The quantum formalism may form a starting point to study quantum effects in the electron gas, such as spinpumping-induced magnetic quantum noise 37 at low temperatures or quantum squeezing and entanglement of the electrons with microwaves via the magnet [38].

The magnetization dynamics expressed by the spin operator $\hat{\mathbf{S}}(\mathbf{r}, t)$ generates a magnetic field by Coulomb's Law 39],

$$
\mathbf{H}_{\beta}(\mathbf{r}, t)=-\frac{\gamma \hbar}{4 \pi} \partial_{\beta} \partial_{\alpha} \int d \mathbf{r}^{\prime} \frac{\left\langle\hat{\mathbf{S}}_{\alpha}\left(\mathbf{r}^{\prime}, t\right)\right\rangle}{\left|\mathbf{r}-\mathbf{r}^{\prime}\right|}
$$

in the summation convention over repeated spatial (or spin) indices $\{\alpha, \beta\}=\{x, y, z\} .-\gamma$ is the gyromagnetic ratio of the nanowire. For sufficiently weak excitation the spin operators in the wire can be expanded into magnon field operators $\hat{\alpha}_{k_{y}}$ and their amplitudes across the nanowire $m_{x, z}^{k_{y}}(x, z)$ :

$$
\hat{\mathbf{S}}_{x, z}(\mathbf{r})=\sqrt{2 S} \sum_{k_{y}}\left(m_{x, z}^{k_{y}}(x, z) e^{i k_{y} y} \hat{\alpha}_{k_{y}}+\text { H.c. }\right)
$$

where $S=M_{s} /(\gamma \hbar)$. The static stray field is negligibly small for sufficiently long nanowires. The dynamic stray field $\mathbf{H}$ is the response to $\left\langle\hat{\alpha}_{k_{y}}\right\rangle$, the coherent amplitude of magnons with momentum $k_{y} \hat{\mathbf{y}}$ excited by external microwaves. With Fourier components $\mathbf{H}_{\beta}(z, \mathbf{k}, t)=$ $\tilde{\mathbf{H}}_{\beta}(z, \mathbf{k}) e^{-i \omega_{\mathrm{K}} t}+\tilde{\mathbf{H}}_{\beta}^{*}(z,-\mathbf{k}) e^{i \omega_{\mathrm{K}} t}$ for $\mathbf{k}=\left(k_{x}, k_{y}, 0\right)^{T}$, below the nanowire $(z<0)$ [17, 22, 23],

$$
\begin{aligned}
\left(\begin{array}{c}
\tilde{\mathbf{H}}_{x}(z, \mathbf{k}) \\
\tilde{\mathbf{H}}_{y}(z, \mathbf{k}) \\
\tilde{\mathbf{H}}_{z}(z, \mathbf{k})
\end{array}\right) & =F_{\mathbf{k}}\left(m_{z}^{k_{y}}+\frac{i k_{x}}{k} m_{x}^{k_{y}}\right)\left(\begin{array}{c}
i k_{x} / k \\
i k_{y} / k \\
1
\end{array}\right) \\
& \times e^{k z}\left|\left\langle\hat{\alpha}_{k_{y}}\right\rangle\right|,
\end{aligned}
$$

where $F_{\mathbf{k}}=-\gamma \hbar \sqrt{2 S}\left(1-e^{-k d}\right) \sin \left(k_{x} w / 2\right) / k_{x}$ is the form factor of the rectangular wire.

$\tilde{\mathbf{H}}(z, \mathbf{k})$ decays exponentially $\sim e^{-k|z|}$ on a scale governed by complex momentum $k_{x} \hat{\mathbf{x}}+k_{y} \hat{\mathbf{y}}-i k \hat{\mathbf{z}}$ [4]. External microwaves excite the Kittel magnon [40] with $k_{y}=0$, such that $\tilde{H}_{y}(\mathbf{k})$ vanishes and $\tilde{H}_{x}(\mathbf{k})=i \operatorname{sgn}\left(k_{x}\right) \tilde{H}_{z}(\mathbf{k})$. The polarization of the Kittel mode is governed by the shape anisotropy and applied magnetic field [41. We focus here on circularly polarized spin waves with $m_{x} \rightarrow i m_{z}$ in nanowires with circular/square cross sections or sufficiently large magnetic field. In this case, $\tilde{\mathbf{H}}(\mathbf{k})=0$ for $k_{x}>0$, since magnons precess preferentially in one direction, which distinguishes the chirality found here from the polarization-momentum locking in optics [2, 3], noting that chirality would vanish for linearly polarized spin waves. The photon spin density under the nanowire $\mathcal{D}(x, z)=\mu_{0} \operatorname{Im}\left[\tilde{\mathbf{H}}^{*}(x, z) \times \tilde{\mathbf{H}}(x, z)\right] /(4 \omega)$ is purely transverse since $\mathcal{D} \cdot \mathbf{H}=0$ for arbitrarily polarized spin waves. As illustrated in Fig. 2 for $w=d=60 \mathrm{~nm}, \mathcal{D}$ is symmetric with respect to the center of the nanowire. At finite distances from the wire the near-singularity at the edges is smeared out, but the average amplitude remains significant. The photon magnetic field couples to the electron spins by the Zeeman interaction. Absorption transfers the photon spin over distances limited by the evanescent decay length in contrast to conventional spin pumping, which happens directly at the interface.

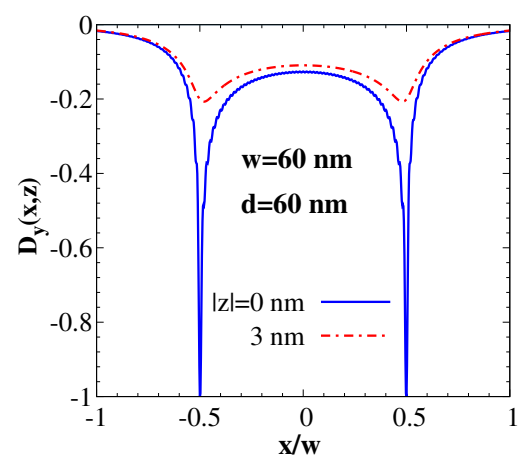

FIG. 2. Spatial distribution of the microwave photon spin density along the $y$-direction (normalized by the maximum modulus when $z=0$ ) generated by a magnetic nanowire with $d=w=60 \mathrm{~nm}$ under FMR. The distances to the nanowire are $z=0$ (solid line) and $3 \mathrm{~nm}$ (dashed-dotted line).

Formalism. - The photon field derived above can excite spins into any conductor in its proximity. Here we illustrate the concept by a $2 \mathrm{DEG}$ with Hamiltonian $\hat{H}_{0}$ in which only the lowest subband with envelope wave function $\phi(z)$ is occupied (see Supplemental Material Sec. III for a 1D quantum wire [42]). The Zeeman coupling between the conduction electron spin $\hat{\mathbf{s}}$ and the evanescent (near) field amplitude $\mathbf{H}(\mathbf{r}, t)$ reads [4, 39]

$$
\hat{H}_{\mathrm{Z}}=\mu_{0} \gamma_{e} \int d \mathbf{r}|\phi(z)|^{2} \hat{\mathbf{s}}(\boldsymbol{\rho}, t) \cdot \mathbf{H}(\mathbf{r}, t),
$$

where $\mathbf{r}=\boldsymbol{\rho}+z \hat{\mathbf{z}}$ and $\gamma_{e}=-g_{e} \mu_{B} / \hbar$ with $\mu_{B}$ and $g_{e}$ being the Bohr magneton and (effective) electron $g$-factor, respectively. In the strictly 2 DEG limit $|\phi(z)|^{2} \rightarrow \delta(z)$ and $\mathbf{H}(\boldsymbol{\rho}, z) \approx \mathbf{H}(\boldsymbol{\rho}, z=0)$. The excited spin density in the linear response reads

$$
s_{l, \alpha}(\boldsymbol{\rho}, t)=-\mu_{0} \gamma_{e} \sum_{\omega, \mathbf{k}} e^{i \mathbf{k} \cdot \boldsymbol{\rho}-i \omega t} \chi_{\alpha \beta}(\mathbf{k}, \omega) H_{\beta}(\mathbf{k}, \omega),
$$

where $\chi_{\alpha \beta}(\mathbf{k}, \omega)$ are the elements of the spin susceptibility tensor [42, 44, 45]. $\mathbf{s}_{l}$ decays with the dipolar field on the scale of the wire width. Assuming that the spin diffusion length, which can be of the order of micrometers in 2DEGs [46] (and even longer in graphene 47]), exceeds the field decay length (tens of nanometer), we can compute $\chi_{\alpha \beta}$ by straightforward linear response theory (see Supplemental Material [42]).

The excited spin density is a source term for the kinetic equations, from which we can calculate spin transport, 
and when the spin-orbit coupling is sufficiently weak, the spin current. For the spin dynamics in the 2DEG we need not only $\mathbf{s}_{l}(\boldsymbol{\rho}, t)$ from Eq. (6), but also its time derivative (" $I$ " for interaction representation) [1, 18, 48 50, which is derived in the Supplemental Material [2]:

$$
\left\langle\frac{\partial \hat{\mathbf{s}}_{I}(\boldsymbol{\rho}, t)}{\partial t}\right\rangle \equiv \boldsymbol{\mathcal { R }}(t)=\frac{\partial \mathbf{s}_{l}(\boldsymbol{\rho}, t)}{\partial t}+\mu_{0} \gamma_{e} \mathbf{s}_{l}(\boldsymbol{\rho}, t) \times \mathbf{H}(\boldsymbol{\rho}, t) .
$$

This relation recovers that in the conventional spin pumping [18, 19] when replacing microwave field $\mathbf{H}$ by the magnetization $\mathbf{m}$ at the interface in Eqs. (6) and (7) [49, in which case $\mathcal{R}(t)$ can be interpreted as the spin injection rate across the interface or spin-current gradient.

When the spin-orbit coupling is negligible, the spin current operator is defined through the commutator $-\nabla_{\boldsymbol{\rho}} \cdot \hat{\mathcal{J}}_{I}=(i / \hbar)\left[\hat{H}_{0}^{I}, \hat{\mathbf{s}}_{I}(\boldsymbol{\rho}, t)\right]$, leading to [48, 49]

$$
-\nabla_{\boldsymbol{\rho}} \cdot \mathcal{J}(\boldsymbol{\rho})=\partial \mathbf{s}_{l}(\boldsymbol{\rho}, t) / \partial t+\mu_{0} \gamma_{e} \mathbf{s}_{l}(\boldsymbol{\rho}, t) \times \mathbf{H}(\boldsymbol{\rho}, t),
$$

where $\mathcal{J}(\boldsymbol{\rho})$ is the spin current tensor with elements $\mathcal{J}_{\alpha}^{\delta}(\boldsymbol{\rho}, z)$ ( $\alpha$ and $\delta$ are the spatial and spin indexes). Substituting Eq. (6) into Eq. (8) leads to DC and AC spin currents. When the susceptibility is well behaved at low frequencies and assuming that the Fermi energy $E_{F} \gg \hbar \omega$, we may use the adiabatic approximation for the excited spin density [44, 48, 51, 52,

$$
\begin{aligned}
& s_{l, \alpha}(\boldsymbol{\rho}, t)=-\mu_{0} \gamma_{e} \sum_{\mathbf{k}} e^{i \mathbf{k} \cdot \boldsymbol{\rho}} \operatorname{Re} \chi_{\alpha \beta}(\mathbf{k}, \omega \rightarrow 0) H_{\beta}(\mathbf{k}, t) \\
& +\left.\mu_{0} \gamma_{e} \sum_{\mathbf{k}} e^{i \mathbf{k} \cdot \boldsymbol{\rho}} \frac{\partial \operatorname{Im} \chi_{\alpha \beta}(\mathbf{k}, \omega)}{\partial \omega}\right|_{\omega \rightarrow 0} \frac{d H_{\beta}(\mathbf{k}, t)}{d t} .
\end{aligned}
$$

In the long wavelength limit in which $\operatorname{Re} \chi_{\alpha \beta}(\mathbf{k} \rightarrow 0, \omega \rightarrow$ 0 ) is constant the first, reactive term on the r.h.s. of Eq. 9 causes a pure $\mathrm{AC}$ contribution $\sim \dot{\mathbf{H}}$ to the spin current through the first term $\dot{\mathbf{s}}$ in the r.h.s. of Eq. (8). The second, dissipative term contributes to a DC spin current. We disregard the nearly homogeneous external microwaves that excites the wire FMR since it does not contribute to the DC response and assume that a small static magnetic field that align the wire magnetization has a negligible effect on the 2DEG spins. The spin current injected under the nanowire can be used as a boundary condition for a spin transport theory [53].

The DC spin current is evaluated below for two model systems with large $g$-factors, viz. the 2DEGs in narrowgap semiconductor heterostructures and topological surface states and for the 1DEG in the Supplemental Material 42 .

Dipolar spin pumping.-For the free electron gas, the spin susceptibility is isotropic [44, 45,

$$
\chi(\mathbf{k}, \omega)=\frac{\hbar^{2}}{2} \sum_{\mathbf{q}} \frac{f\left(\xi_{\mathbf{q}}\right)-f\left(\xi_{\mathbf{k}+\mathbf{q}}\right)}{\hbar \omega+i 0_{+}+\xi_{\mathbf{q}}-\xi_{\mathbf{k}+\mathbf{q}}},
$$

where $\xi_{\mathbf{k}}=\hbar^{2} k^{2} /\left(2 m^{*}\right)-\mu$ is the electron energy with effective mass $m^{*}$, relative to the chemical potential $\mu$, and $f\left(\xi_{\mathbf{k}}\right)=\left\{\exp \left[\xi_{\mathbf{k}} /\left(k_{B} T\right)\right]+1\right\}^{-1}$ is the Fermi-Dirac distribution at temperature $T$. In the microwave regime for the nanowire, $\left|k_{x}\right|<2 k_{F}$, where $k_{F}$ is the 2DEG Fermi vector [in semiconductors $k_{F}=O\left(\mathrm{~nm}^{-1}\right)$ ] and $\operatorname{Re} \chi\left(\left|k_{x}\right|, \omega \rightarrow 0\right)=m^{*} /\left(\pi \hbar^{2}\right)$ [45] and the reactive first term vanishes. The DC spin current then reduces to

$$
\begin{aligned}
\mathcal{J}_{x}^{\mathrm{DC}}(x) & =\left.\left(\mu_{0} \gamma_{e}\right)^{2} \int_{0}^{x} d x \sum_{k_{x}} e^{i k_{x} x} \partial_{\omega} \operatorname{Im} \chi\left(\left|k_{x}\right|, \omega\right)\right|_{\omega=0} \\
& \times\left\langle\dot{\mathbf{H}}\left(k_{x}, t\right) \times \mathbf{H}(x, t)\right\rangle_{\mathrm{DC}},
\end{aligned}
$$

where we used the symmetry relations $\mathcal{J}_{x}^{\mathrm{DC}}\left(k_{x}\right)=$ $-\mathcal{J}_{x}^{\mathrm{DC}}\left(-k_{x}\right)$ and $\mathcal{J}_{x}^{\mathrm{DC}}(x=0)=0$ [42]. Assuming for the moment that $\chi\left(\left|k_{x}\right|, \omega\right) \approx \chi\left(k_{\text {ave }}, \omega\right)$ with $k_{\text {ave }} \sim \pi /(2 w)$, we obtain the simplified expression at FMR

$$
\begin{aligned}
\mathcal{J}_{x}^{\mathrm{DC}}(x) & \approx-\left.2\left(\mu_{0} \gamma_{e}\right)^{2} \omega_{\mathrm{K}} \partial_{\omega} \operatorname{Im} \chi\left(\left|k_{x}\right| \rightarrow k_{\text {ave }}, \omega\right)\right|_{\omega=0} \\
& \times \int_{0}^{x} d x^{\prime} \operatorname{Im}\left[\tilde{\mathbf{H}}^{*}\left(x^{\prime}\right) \times \tilde{\mathbf{H}}\left(x^{\prime}\right)\right] .
\end{aligned}
$$

Hence, the DC spin-current below the nanowire is (approximately) proportional to the transverse spin of the magnetic field, implying transfer of the photon spin angular momentum to the electron spin with an efficiency governed by $\left.\partial_{\omega} \operatorname{Im} \chi\left(\left|k_{x}\right| \rightarrow k_{\text {ave }}, \omega\right)\right|_{\omega=0}$. The spin current is polarized in the $-y$-direction, i.e., opposite to the magnetization direction of the nanowire.

Since $\mathcal{J}_{x}(x) \propto \operatorname{sgn}(x)$, the excited spin is not chiral, but flows into both directions on both sides of the nanowire as indicated by the blue arrow in Fig. 1, just as in conventional spin pumping [18, 48, 51, 52. Although excited by the same field, this result is in stark contrast to the magnon spin current [11, 17, 22, 23] or the chiral energy currents of surface plasmon polaritons excited by a rotating electric dipole [5, 6, 8, which are both unidirectional and flow in half space. We can trace the different physics to the collective nature of magnons/plasmons with a well-defined dispersion relation that in the present geometry are symmetric in $k$-space, but of which a chiral dipolar field selects only one. The susceptibility of the non-interacting electron gas, on the other hand, is made up by a broad spectrum of electron-hole pair excitations at the Fermi energy, and chirality vanishes in the integral over wave vectors at a given frequency. Interestingly, chirality emerges for metallic carbon nanotubes or interacting electrons in a quantum wire that cross the magnetic wire at right angles [42, because the spin susceptibility in the $1 \mathrm{DEG}$ with a linear dispersion at the Fermi energy such as metallic carbon nanotubes with (Tomonaga-Luttinger liquid) or without interactions is singular [42, 44]

$$
\chi(k, \omega)=\frac{\hbar k L}{2 \pi}\left(\frac{1}{\omega+i 0_{+}-v_{F} k}+\frac{1}{\omega+i 0_{+}+v_{F} k}\right),
$$


where $L$ is the system length and $v_{F}$ is the Fermi velocity. By contour integration and the chiral dipolar field with $\mathbf{H}\left(k_{x}>0, \omega_{\mathrm{K}}\right)=0$ for the right-circularly polarized spin waves,

$$
\mathbf{s}_{l}(x, t)=\operatorname{Im}\left(-\frac{2 \mu_{0} \gamma_{e} \omega_{\mathrm{K}}}{v_{F}^{2}} \mathbf{H}\left(k_{-}, \omega_{\mathrm{K}}\right) e^{i k_{-} x-i \omega_{\mathrm{K}} t}\right),
$$

when $x<0$ but vanishes when $x>0$, with $k_{-}=$ $-\omega_{\mathrm{K}} / v_{F}$, implying that the excited spin density lives only in half of the nanowire. The DC spin current vanishes when $x>0$, but flows in the same half space $x<0$ with

$$
\mathcal{J}_{x}(x, t)=-\mu_{0} \gamma_{e} \int_{0}^{x} d x^{\prime} \mathbf{s}_{l}\left(x^{\prime}, t\right) \times\left.\mathbf{H}\left(x^{\prime}, t\right)\right|_{\mathrm{DC}},
$$

recovering the chiral excitation of a spin-density current [42] found earlier in magnetic films. This example proves that quite generally chiral excitation by dipolar radiation is not caused by a hidden symmetry, but requires poles in the spin-susceptibility generated by degenerate electronhole pairs, or the plasmon, magnon, phonon excitations of a rigid ground state.

We now estimate the magnitude of the DC spin current and/or spin-injection rate by the dipolar field from an excited magnetic nanowire. We choose a symmetric QW with $s=20 \mathrm{~nm}$ of a semiconductor with small effective mass such as InSb with $m^{*}=0.015 m_{e}$ 31 and electron density $n_{e}=3 \times 10^{11} \mathrm{~cm}^{-2}$ (corresponding to a Fermi energy $E_{F} \sim 50 \mathrm{meV}$ and Fermi temperature $560 \mathrm{~K})$, such that only the lowest band is populated even at room temperature. The Dresselhaus-type spinorbit coupling with coefficient $\gamma_{D}=220 \mathrm{eVA}^{3}$ [31, 54 causes a small correction $\gamma_{D}(\pi / s)^{2} k_{F} \sim 0.7 \mathrm{meV} \ll E_{F}$ that we disregard. The $g$-factor of electron is $g_{e}=-36$ [31, 32, but the sign is not important here. At temperature $T=100 \mathrm{~K}$ the system is degenerate with subband splitting $\hbar^{2}(\pi / s)^{2} /\left(2 m^{*}\right)=63 \mathrm{meV} \gg k_{B} T$. For a $\mathrm{Co}$ or $\mathrm{CoFeB}$ nanowire with $w=d=60 \mathrm{~nm}$ and $\mu_{0} M_{s}=1.2 \mathrm{~T}[23$, we assume a coherent magnon density $\rho_{m}=\left|\left\langle\hat{\alpha}_{k_{y}=0}\right\rangle\right|^{2}=10^{9}$ in Eq. (3) that corresponds to a transverse magnetization amplitude $M_{x, y} \sim$ $2 \sqrt{2 \gamma \hbar M_{s} \rho_{m}} m_{x, y}^{k_{y}=0}$, i.e. a small precession cone angle $\sim 3.2 \times 10^{-3}$ degrees that is easily excited by FMR.

We plot the DC spin current under the nanowire from Eqs. (11) and 12 in Fig. 3(a), in which the bidirectional spin current is indicated by the black arrows, and the spin-injection rate in Fig. 3(b). The simplified Eq. 12 describes the pumped current by Eq. (11) well. With the same conditions, the pumped spin current is four times in magnitude smaller in InAs QWs $\left(m^{*}=0.023 m_{e}\right.$ and $\left|g_{e}\right|=14.3$ [31]). The spin current is of the same order as the spin Hall current generated by an electric field of $0.1 \mathrm{kV} / \mathrm{cm}$ and a spin Hall conductivity $\sigma_{x}^{y}=10^{6}(\Omega \cdot \mathrm{m})^{-1}$, which should be easily measurable [55]. Under the same conditions, the spin current pumped by the dipolar interaction is comparable with that from interfacial exchange interaction with an exchange splitting $J M_{s} \hbar \sim 10 \mathrm{meV}$ [56], but does not require good electric contact between magnet and semiconductor.
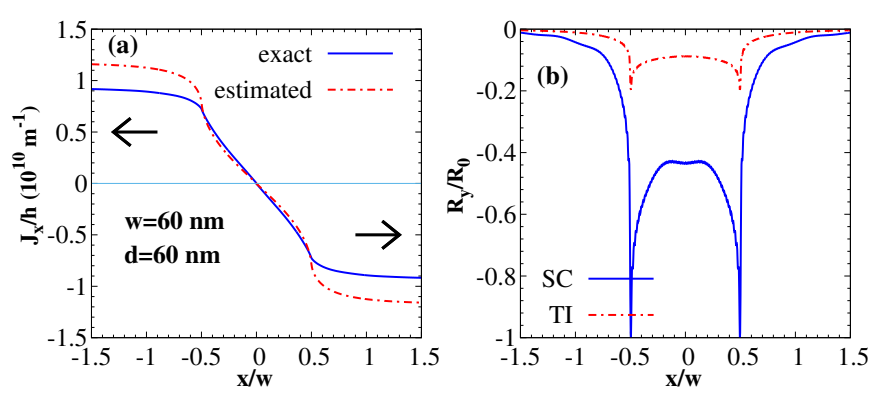

FIG. 3. Excited spin current $\mathcal{J}_{x} / h$, with $h=2 \pi \hbar[(\mathrm{a})]$ and spin-injection rate $[(b)]$ under an excited Co nanowire. In (a), the blue and red curves are calculated from Eqs. (11) and $\sqrt{12}$, respectively. In (b), the blue and red curves are the spin injection rates for a semiconductor 2DEG and the surface state of a topological insulator, normalized by the maximal magnitude $R_{0} / \hbar=2.7 \times 10^{18} \mathrm{~m}^{-2}$ of the blue curve.

The excited spin current under the transducer drives diffusive spin transport over the spin diffusion length scale [53]. The spin signal can be converted to a transverse voltage by the inverse spin Hall effect in the 2DEG itself or by heavy metal contacts [55] or the inverse Edelstein effect [57]. The cyclotron resonance excited by evanescent microwave magnetic fields in the quantum Hall regime could be an interesting extension of the present work.

Finally, we estimate the efficiency of the dipolar spin pumping for surface states of the $n$-doped topological insulator $\mathrm{Bi}_{2} \mathrm{Se}_{3}[58$ at a low temperature $T=30 \mathrm{~K}$ for which a good exchange interaction with magnetic contacts is difficult to achieve 34] and perhaps not desired because of an associated proximity effect. Since the spin current is not conserved, we focus on the DC spin injection rate $\mathcal{R}$ defined in Eq. (7) and compared with the semiconductor case in Fig. 3(b). Only the diagonal terms of the susceptibility tensor 59 ]

$$
\chi\left(\left|k_{x}\right|, \omega\right)=\frac{\hbar^{2}}{8} \sum_{\mathbf{q}}\left(1-\cos \phi_{\mathbf{q}}\right) \frac{n_{F}\left(\xi_{\mathbf{q}-\mathbf{k}}\right)-n_{F}\left(\xi_{\mathbf{q}}\right)}{\hbar \omega+i 0_{+}+\xi_{\mathbf{q}-\mathbf{k}}-\xi_{\mathbf{q}}},
$$

where $\xi_{\mathbf{k}}=\hbar v_{F} k-\mu$, contribute to the DC spin injection (see Supplemental Material [42]). With $n_{e}=10^{11} \mathrm{~cm}^{-2}$, $v_{F}=10^{5} \mathrm{~m} / \mathrm{s}$, and $\left|g_{e}\right|=20[33$, the spin injection rate is of the same order as that of InSb semiconductor 2DEG.

Discussion.- Spin pumping by evanescent microwaves is a coherent mechanism for the generation of pure spin currents in conventional spintronic systems and devices that requires nanomagnets rather than extended films. The effect is not small: spin currents generated by the stray fields of Co nanowires on top of a thin yttrium iron 
garnet film exceed those by the exchange coupling [23]. Here we focus on transverse spin pumping into a conductor, which is most efficient for low-dimensional electron systems. We predict here a transverse spin current density $10^{-13} \mathrm{~J} / \mathrm{m}$ pumped into an InAs 2DEG, which is almost two orders of magnitude larger than what has been observed for the spin-pumping by a Py slab into graphene [29, whose signal should indeed be much smaller with small $g$-factor. We therefore cannot exclude that the observations are caused by dipolar fields at the edge of $\mathrm{Py}$, and not exchange interactions at interface. In general, however, spin pumping into 2DEGs by extended magnetic films 60 63. (and in basically all planar structures used in conventional spin pumping experiments) should be dominated by the exchange mechanism.

The photon angular momentum is inherent to the evanescent stray fields of a precessing magnetization, but it also exists in microwave cavities or waveguides. The dipolar spin pumping is contactless and avoids possible artifacts by the magnetic proximity effect. The excited spin current is not chiral for 2DEGs, but chirality reemerges in the 1DEG. The spin pumping by a magnetic transducer into a 2DEG and a surface state of a topological insulator are estimated large enough to be observable. Our study bridges the concepts and understandings in different fields including spintronics [1, 12, 13, 19, 64, nano-optics [5] and plasmonics [2, 3].

This work is financially supported by the Nederlandse Organisatie voor Wetenschappelijk Onderzoek (NWO) as well as JSPS KAKENHI Grant No. 19H006450. We thank Yaroslav Tserkovnyak, Alejandro O. Leon and Jin Lan for useful discussions.

[1] S. D. Bader and S. S. P. Parkin, Annu. Rev. Condens. Matter Phys. 1, 71 (2010).

[2] K. Y. Bliokh, D. Smirnova, and F. Nori, Science 348, 1448 (2015).

[3] K. Y. Bliokh and F. Nori, Phys. Rep. 592, 1 (2015).

[4] J. D. Jackson, Classical Electrodynamics, (Wiley, New York, 1998).

[5] L. Novotny and B. Hecht, Principles of Nano-Optics (Cambridge University Press, Cambridge, England, 2006).

[6] F. J. Rodríguez-Fortuño, G. Marino, P. Ginzburg, D. O'Connor, A. Martínez, G. A. Wurtz, and A. V. Zayats, Science 340, 328 (2013).

[7] T. Van Mechelen and Z. Jacob, Optica 3, 118 (2016).

[8] J. Petersen, J. Volz, and A. Rauschenbeutel, Science 346, 67 (2014).

[9] T. Schneider, A. A. Serga, T. Neumann, B. Hillebrands, and M. P. Kostylev, Phys. Rev. B 77, 214411 (2008).

[10] V. E. Demidov, M. P. Kostylev, K. Rott, P. Krzysteczko, G. Reiss, and S. O. Demokritov, Appl. Phys. Lett, 95, 2509 (2009).

[11] T. Yu and G. E. W. Bauer, in Chirality, magnetism, and magnetoelectricity: Separate phenomena and joint effects in metamaterial structures, Edited by E. Kamenetskii (Springer, unpublished), arXiv:2001.06821.

[12] B. Lenk, H. Ulrichs, F. Garbs, and M. Muenzenberg, Phys. Rep. 507, 107 (2011).

[13] A. V. Chumak, V. I. Vasyuchka, A. A. Serga, and B. Hillebrands, Nat. Phys. 11, 453 (2015).

[14] D. Grundler, Nat. Nanotechnol. 11, 407 (2016).

[15] V. E. Demidov, S. Urazhdin, G. de Loubens, O. Klein, V. Cros, A. Anane, and S. O. Demokritov, Phys. Rep. 673, 1 (2017).

[16] M. Büttiker, H. Thomas, and A. Prêtre, Z. Phys. B 94, 133 (1994).

[17] T. Yu, Y. M. Blanter, and G. E. W. Bauer, Phys. Rev. Lett. 123, 247202 (2019).

[18] Y. Tserkovnyak, A. Brataas, and G. E. W. Bauer, Phys. Rev. Lett. 88, 117601 (2002).

[19] Y. Tserkovnyak, A. Brataas, G. E. W. Bauer, and B. I. Halperin, Rev. Mod. Phys., 77, 1375 (2005).

[20] J. L. Chen, C. P. Liu, T. Liu, Y. Xiao, K. Xia, G. E. W. Bauer, M. Z. Wu, and H. M. Yu, Phys. Rev. Lett. 120, 217202 (2018).

[21] Y. Au, E. Ahmad, O. Dmytriiev, M. Dvornik, T. Davison, and V. V. Kruglyak, Appl. Phys. Lett. 100, 182404 (2012).

[22] T. Yu, C. P. Liu, H. M. Yu, Y. M. Blanter, and G. E. W. Bauer, Phys. Rev. B 99, 134424 (2019).

[23] J. L. Chen, T. Yu, C. P. Liu, T. Liu, M. Madami, K. Shen, J. Y. Zhang, S. Tu, M. S. Alam, K. Xia, M. Z. Wu, G. Gubbiotti, Y. M. Blanter, G. E. W. Bauer, and H. M. Yu, Phys. Rev. B 100, 104427 (2019).

[24] T. Yu, Y.-X. Zhang, S. Sharma, X. Zhang, Y. M. Blanter, and G. E. W. Bauer, Phys. Rev. Lett. 124, 107202 (2020).

[25] T. Yu, X. Zhang, S. Sharma, Y. M. Blanter, and G. E. W. Bauer, Phys. Rev. B 101, 094414 (2020).

[26] M. Grassi, M. Geilen, D. Louis, M. Mohseni, T. Brächer, M. Hehn, D. Stoeffler, M. Bailleul, P. Pirro, and Y. Henry, arXiv:1912.09735.

[27] J. B. S. Mendes, O. A. Santos, L. M. Meireles, R. G. Lacerda, L. H. Vilela-Leão, F. L. A. Machado, R. L. Rodríguez-Suárez, A. Azevedo, and S. M. Rezende, Phys. Rev. Lett. 115, 226601 (2015).

[28] Z. Tang, E. Shikoh, H. Ago, K. Kawahara, Y. Ando, T. Shinjo, and M. Shiraishi, Phys. Rev. B 87, 140401(R) (2013).

[29] D. Indolese, S. Zihlmann, P. Makk, C. Jünger, K. Thodkar, and C. Schönenberger, Phys. Rev. App. 10, 044053 (2018).

[30] T. Inoue, G. E. W. Bauer, and K. Nomura, Phys. Rev. B 94, 205428 (2016).

[31] J. M. Jancu, R. Scholz, E. A. de Andrada e Silva, and G. C. La Rocca, Phys. Rev. B 72, 193201 (2005).

[32] Semiconductors, edited by O. Madelung, LandoltBörnstein, New Series Vol. 17a (Springer, Berlin, 1987).

[33] A. Wolos, S. Szyszko, A. Drabinska, M. Kaminska, S. G. Strzelecka, A. Hruban, A. Materna, M. Piersa, J. Borysiuk, K. Sobczak, and M. Konczykowski, Phys. Rev. B 93, 155114 (2016).

[34] Y. T. Fanchiang, K. H. M. Chen, C. C. Tseng, C. C. Chen, C. K. Cheng, S. R. Yang, C. N. Wu, S. F. Lee, M. Hong, and J. Kwo, Nat. Commun. 9, 223 (2018).

[35] H. Yu, G. Duerr, R. Huber, M. Bahr, T. Schwarze, F. Brandl, and D. Grundler, Nat. Commun. 4, 2702 (2013). 
[36] C. P. Liu, J. L. Chen, T. Liu, F. Heimbach, H. M. Yu, Y. Xiao, J. F. Hu, M. C. Liu, H. C. Chang, T. Stueckler, S. Tu, Y. G. Zhang, Y. Zhang, P. Gao, Z. M. Liao, D. P. Yu, K. Xia, N. Lei, W. S. Zhao, and M. Z. Wu, Nat. Commun. 9, 738 (2018).

[37] C. Gardiner and P. Zoller, Quantum Noise (SpringerVerlarg, Berlin, 2000).

[38] J. Zou, S. K. Kim, and Y. Tserkovnyak, Phys. Rev. B 101, 014416 (2020).

[39] L. D. Landau and E. M. Lifshitz, Electrodynamics of Continuous Media, 2nd ed. (Butterworth-Heinenann, Oxford, 1984).

[40] C. Kittel, Phys. Rev. 73, 155 (1948).

[41] For the Kittel mode, $m_{x}=i \sqrt{\mathcal{D} /(4 w d)}$ and $m_{z}=$ $\sqrt{1 /(4 \mathcal{D} w d)}$, where $\mathcal{D}=\sqrt{\frac{H_{0}+N_{z z} M_{s}}{H_{0}+N_{x x} M_{s}}}$ is determined by the applied field $H_{0}$ and $M_{s}$ with $N_{x x} \simeq d /(d+w)$ and $N_{z z} \simeq w /(d+w)$. It is circularly polarized $m_{z}=i m_{x}$ at large magnetic fields $\left(H_{0} \gg M_{s}\right)$ and/or when the wire has a square cross section. It tends to be linearly polarized at weak applied fields and when $w \gg d$.

[42] See Supplemental Material [...] for the derivation of spin injection rate, spin susceptibility and the spin current at origin $\mathcal{J}_{x}(x=0)$, which includes Refs. [18, 43, 45, 49, 58, 59 .

[43] J. Shi, P. Zhang, D. Xiao, and Q. Niu, Phys. Rev. Lett. 96, 076604 (2006).

[44] G. D. Mahan, Many Particle Physics (Plenum, New York, 1990).

[45] G. F. Giuliani and G. Vignale, Quantum Theory of the Electron Liquid (Cambridge University Press, Cambridge, 2005).

[46] G. Wang, B. L. Liu, A. Balocchi, P. Renucci, C. R. Zhu, T. Amand, C. Fontaine, and X. Marie, Nat. Commun. 4, 2372 (2013).

[47] J. Ingla-Aynés, M. H. D. Guimarães, R. J. Meijerink, P. J. Zomer, and B. J. van Wees, Phys. Rev. B 92, 201410(R) (2015).

[48] A. B. Cahaya, A. O. Leon, and G. E. W. Bauer, Phys. Rev. B 96, 144434 (2017).

[49] Y. Ohnuma, H. Adachi, E. Saitoh, and S. Maekawa,
Phys. Rev. B 89, 174417 (2014).

[50] T. Yu and M. W. Wu, Phys. Rev. B 89, 045303 (2014).

[51] E. Šimánek and B. Heinrich, Phys. Rev. B 67, 144418 (2003).

[52] E. Šimánek, Phys. Rev. B 68, 224403 (2003).

[53] T. Yu and M. W. Wu, Phys. Rev. A 92, 013607 (2015).

[54] A. N. Chantis, M. van Schilfgaarde, and T. Kotani, Phys. Rev. Lett. 96, 086405 (2006).

[55] J. Sinova, S. O. Valenzuela, J. Wunderlich, C. H. Back, and T. Jungwirth, Rev. Mod. Phys. 87, 1213 (2015).

[56] The linearized interface exchange Hamiltonian $\hat{H}_{\mathrm{ex}}=$ $J \int \hat{\mathbf{s}}(\boldsymbol{\rho}, t) \cdot \hat{\mathbf{M}}(\mathbf{r}, t) d \boldsymbol{\rho}$, with $\hat{\mathbf{M}} \simeq\left(m_{x}(x), M_{s}, m_{z}(x)\right)^{T}$. With $\mathbf{m}(x, t)=\tilde{\mathbf{m}}\left(x, \omega_{\mathrm{K}}\right) e^{-i \omega_{\mathrm{K}} t}+\tilde{\mathbf{m}}^{*}\left(x, \omega_{\mathrm{K}}\right) e^{i \omega_{\mathrm{K}} t}$, we arive at the estimate

$$
\begin{aligned}
\mathcal{J}_{x}^{\mathrm{DC}}(x) & \approx-\left.2 J^{2} \omega_{\mathrm{K}} \partial_{\omega} \operatorname{Im} \chi\left(\left|k_{x}\right| \rightarrow \pi /(2 w), \omega\right)\right|_{\omega=0} \\
& \times \int_{0}^{x} d x^{\prime} \operatorname{Im}\left[\tilde{\mathbf{m}}^{*}\left(x^{\prime}\right) \times \tilde{\mathbf{m}}\left(x^{\prime}\right)\right]
\end{aligned}
$$

[57] J. C. R. Sánchez, L. Vila, G. Desfonds, S. Gambarelli, J. P. Attané, J. M. De Teresa, C. Magén, and A. Fert, Nat. Commun. 4, 2944 (2013).

[58] Y. Xia, D. Qian, D. Hsieh, L. Wray, A. Pal, H. Lin, A. Bansil, D. Grauer, R. J. Cava, and M. Z. Hasan, Nat. Phys. 5, 398 (2009).

[59] J. H. Jiang and S. Wu, Phys. Rev. B 83, 205124 (2011).

[60] K. Ando, S. Takahashi, J. Ieda, H. Kurebayashi, T. Trypiniotis, C. H. W. Barnes, S. Maekawa, and E. Saitoh, Nat. Mat. 10, 655 (2011).

[61] Q. Song, H. Zhang, T. Su, W. Yuan, Y. Chen, W. Xing, J. Shi, J. Sun, and W. Han, Sci. Adv. 3, e1602312 (2017).

[62] Y. T. Fanchiang, K. H. M. Chen, C. C. Tseng, C. C. Chen, C. K. Cheng, S. R. Yang, C. N. Wu, S. F. Lee, M. Hong, and J. Kwo, Nat. Commu. 9, 223 (2018).

[63] H. L. Wang, J. Kally, C. Sahin, T. Liu, W. Yanez, E. J. Kamp, A. Richardella, M. Z. Wu, M. E. Flatté, and N. Samarth, Phys. Rev. Res. 1, 012014(R) (2019).

[64] I. Žutić, J. Fabian, and S. Das Sarma, Rev. Mod. Phys. 76, 323 (2004). 